\title{
Glotopolíticas literarias entre resistencia y mercado: Bolaño en traducción, la traducción en Bolaño
}

\section{Literatura mundial y/en traducción: de ganancias, pérdidas y traductores (in-)visibles}

Afirmar que el acto de traducir es el sine qua non de la existencia de la literatura mundial es una evidencia ya destacada por Goethe en uno de sus tantos pasajes acerca del tema, ya que "diga lo que se diga acerca de la insuficiencia de la traducción, es y será uno de los negocios más importantes y dignos del tráfico mundial general" (434) ${ }^{1}$. Al mismo tiempo -y en concordancia con la afirmación del clásico alemán- la traducción ocupa un lugar ambiguo en la amplia tradición de su investigación teórica: es indispensable para la circulación de textos, ideas y como sugiere la dominante metáfora economicista del Verkehr en las nociones de Goethe- de mercancías, sin que por ello pudiese superar el eterno defecto de su propia imperfectibilidad. "Nothing is translatable. [...] Everything is translatable" es la fórmula paradójica que Emily Apter ha usado en los debates contemporáneos para designar los dos "poles of translation theory" (The Tranlation Zone 8), entre los que se podría situar gran parte de los textos teóricos recientes. Uno de los ejemplos más prominentes resulta ser la reconocida (re-)definición de la literatura mundial por parte de David Damrosch, quien postula que esta sería, principalmente, "writing that gains in translation" (281). Si bien, al adherir más bien a un lenguaje metafórico ${ }^{2}$-“works become world literature when they gain

1 Traducción propia del original ("Denn was man auch von der Unzulänglichkeit des Übersetzens sagen mag, so ist und bleibt es doch eines der wichtigsten und würdigsten Geschäfte in dem allgemeinen Weltverkehr"). Acerca del aspecto de la traducción en Goethe, véanse también las reflexiones de Strich (15-30). Sobre la importancia de los estudios de traducción en el debate actual sobre literatura mundial, véase el artículo de Venuti que parte de la afirmación: "World Literature cannot be conceptualized apart from translation" ("World Literature" 180).

2 Véanse al respecto las reflexiones de Harrison y su crítica de que "the fundamental problem with Damrosch's assertion about gains in translation is that it seems to assume arbitrarily that gains outweigh losses” (415).

Benjamin Loy, Universität zu Köln

Ә Open Access. () 2020 Benjamin Loy, published by De Gruyter. (cc) BY-NC-ND This work is licensed under a Creative Commons Attribution-NonCommercial-NoDerivatives 4.0 International License.

https://doi.org/10.1515/9783110673678-014 
on balance in translation, stylistic losses offset by an expansion in depth as they increase their range" (289)-, Damrosch no profundiza realmente en las dimensiones materiales del negocio translaticio, subraya la importancia de la traducibilidad de una obra como factor para su circulación internacional, ya que "some works are not translatable without substantial loss, and so they remain largely within their local or national context, never achieving an effective life as world literature" (288-289). Frente a esa larga lista de posiciones de una Weltliteraturwissenschaft (22) (como la ha llamado Fritz Strich) que explican "why translation matters" (Grossman), siendo la base de una literatura mundial entendida como un conjunto de "multiple windows on the world" (Damrosch 15), el polo opuesto del espectro teórico ha preferido insistir en las dimensiones problemáticas de la traducción: aparte de complejizar la noción del fenómeno en el sentido de una "política de la traducción” (Spivak, “The Politics”), implicando también una problematización del acto de traducir como tal que contribuye -como es el caso en gran parte del mercado literario y universitario global ${ }^{3}$ - a una homogenización lingüística (Spivak, Death of a discipline), esos trabajos han llamado la atención sobre aspectos de "intraducibilidad" como opuesto crítico y productivo ${ }^{4}$ del discurso translaticio afirmativo.

El punto en común de ese tipo de trabajos reside en sus perspectivas teóricas y muchas veces abstractas frente a ejemplos de traducciones literarias concretas. Esto conduce -siguiendo la separación entre traductología y práctica traslaticia establecida por Antoine Berman (69) - a la pregunta por la importancia de una crítica de la traducción enfocada en el texto concreto, es decir en la dimensión filológica y -en el mejor de los sentidos-artesanal que representa el centro imprescindible de cualquier trabajo translaticio como operación textual ${ }^{5}$. La necesidad de semejante trabajo crítico se hace más evidente si se considera, además, el papel que ocupan tanto la traducción como las traductoras en la crítica literaria actual. A diferencia de la "invisibilidad" de la traductora, como lo formuló de manera prominente Lawrence Venuti en sus críticas a la “illusion of transparency” (1)

3 Walkowitz destaca la velocidad y simultaneidad de la circulación internacional contemporánea: "The translation and circulation of literature today is historically unprecedented once we consider how quickly books enter various national markets, small and large, across several continents" (2).

4 Apter hace hincapié en ese aspecto al entender lo intraducible "not as pure difference in opposition to the always translatable (rightly suspect as just another non-coeval form of the romantic Absolute, or fetish of the Other, or myth of hermeneutic inaccessibility) but as a linguistic form of creative failure with homeopathic uses" (Against World Literature 20).

5 , para decirlo en palabras de uno de los precursores de una filología de la literatura mundial, "El arte de la interpretación está perdido si se cree poder descuidar las palabras claras del texto en nombre de una idea poética mayor" (Auerbach 141). 
que ha imperado tradicionalmente en el mercado literario anglo-americano, en los últimos años una nueva tendencia (sobre cuya magnitud ciertamente se puede discutir) parece haber surgido en las editoriales y en los suplementos culturales de los principales medios para hacer frente al trato y la presencia de las traducciones y las traductoras: así como (al menos algunas de) las editoriales optan por una nueva visibilidad de las autoras de la traducción de una obra (a través de su presencia en las portadas de los libros, por ejemplo), así también cada día son más numerosas las reseñas literarias de obras traducidas que -en una especie de acto de political correctness literaria prácticamente internalizado- reservan una frase obligatoria para expresar la calidad (casi siempre positiva) de la labor translaticia en cuestión. Sin embargo, por más que se quiera valorar esta nueva sensibilidad de la crítica frente al oficio de las traductoras que, por otro lado, siguen siendo (con pocas excepciones) “the literary proletariat” (Apter, The Translation Zone xi), no caben dudas de que dicho discurso reconocedor se reduce en la mayoría de los casos a una fórmula vacía ya que los juicios emitidos sobre la calidad de una traducción no explican ni remotamente sus motivos en concordancia con un conocimiento del original. Como anota Walkowitz, "We think about writing all the time, of course, but whenever we talk about translation we seem to know and to agree what a translator translates from" (173). De esta manera, la supuesta atención lograda por la traducción -al menos con respecto a los medios dominantes en Europa y América ${ }^{6}$ - termina siendo una operación que sustituye la "ilusión de la transparencia” y la negación del carácter translaticio de una obra (en el sentido de Venuti) por una especie de "ilusión de la visibilidad": la traducción es valorada porque el acto de traducir como tal forma parte del discurso y de las prácticas cosmopolitas contemporáneos, sin que eso signifique una reflexión sobre el problema de cómo encontrar en reseñas literarias y otros formatos no-académicos "an intelligent way to review both the original and its translation within the space limitations imposed by the publication" (Grossmann 32). Si Grossman tilda ese tipo de falencias en la prensa cultural como "product of intransigent dilettantism and tenacious amateurism" (32), cabe aquí subrayar el hecho de que la vasta producción de trabajos académicos dedicados al paradigma de World Literature (y el papel de la traducción) en su gran mayoría ha omitido la dimensión de una crítica concreta textual y/o comparativa- de obras en su “vida en traducción”. Por tal motivo, en

\footnotetext{
6 En el mundo cibernético la situación -como se verá más adelante- difiere de manera significativa con la de la prensa escrita al existir una multitud de revistas y blogs en línea dedicados a la traducción, la crítica de la traducción, la presentación de traductoras contemporáneas, etc. (véanse también algunos ejemplos en Walkowitz 235-245). De la misma manera habría que diferenciar, sin lugar a dudas, entre la crítica de novelas y de poesía, siendo esta última (y por razones obvias) mucho más sensible frente a las dimensiones concretas de obras en traducción.
} 
lo que sigue este artículo adhiere claramente a la posición (y la metodología) de Venuti con respecto a la relación entre literatura mundial y traducción:

Clearly, the formal and semantic gain that enables translation to define world literature cannot be perceived without close reading, without a detailed analysis that examines shifts between the source and translated texts. "Distant" reading [...] is essential to understanding world literature as an intricate, historically developing ensemble of crosscultural relations among major and minor traditions [...]. Yet allowing the text to disappear as a unit of analysis between these two extremes would be counterproductive: the text not only links the small feature and the large structure, showing how they depend on one another for their literary and cultural significance, but it also makes visible the role of translation in the construction of world literature. ("World Literature" 186)

En este sentido, un ejemplo paradigmático lo constituye la obra de Roberto Bolaño, que, debido a su enorme éxito y circulación mundial, se analizará a continuación como un "estudio de caso" en el contexto de la problemática esbozada hasta aquí. Partiendo de una breve discusión de los trabajos críticos dedicados al aspecto de la traducción y recepción mundial de Bolaño, se discutirá, con base en un análisis comparativo puntualmente de las traducciones de su novela Estrella distante al inglés, alemán y francés, la paradójica hipótesis de que, por una parte, la idea de la traducción y de la traducibilidad es fundamental en la obra de Bolaño pero que posee, al mismo tiempo, solamente una importancia secundaria para la obra en cuanto texto traducido en circulación internacional y como parte de una literatura mundial contemporánea. Metodológicamente el trabajo entonces hace énfasis tanto en la práctica (muchas veces descuidada) de un close reading comparativo de traducciones (siguiendo a Venuti y recurriendo sobre todo a algunos aspectos de los trabajos traductológicos de Berman) como en la dimensión más bien "sociológica” (o de un distant reading) de los distintos factores de recepción y circulación global de la obra de Bolaño para desarrollar, de esta manera, una perspectiva más amplia sobre la relación entre traducción y literatura mundial ${ }^{7}$.

7 Retomo parcialmente la propuesta de Walkowitz, quien insiste en una combinación de "close reading, book history, and translation studies" (83) y una renovada atención global "to all aspects of the literary work [...] [to] conceive of details more broadly and more variously as those parts of the text, of potentially any scale or size, that seem prosaic, beneath notice, or simply mechanical" (90). Walkowitz también integra, como una de las pocas críticas del debate actual, un pequeño estudio comparativo de traducciones dedicado a la obra That's how you lose her, de Junot Díaz (35-39). De manera parecida, Venuti sostiene en la necesidad “[to] combine distant and close reading of translation to explore the relations between canons and interpretations" ("World Literature” 191). 


\section{Bolaño en traducción: posiciones y equívocos}

Una prueba del importante lugar que ocupa la obra de Roberto Bolaño dentro de los recientes debates en torno a la literatura mundial ${ }^{8}$ (y el papel de la traducción en especial) es su inserción dentro de algunas de las contribuciones más importantes (y ya nombradas) de la literatura comparada al respecto: así, por ejemplo, Walkowitz incluye Los detectives salvajes en sus reflexiones sobre lo que denomina "born-translated literature" al argüir que "[h]is novels seem translated, in part because they combine several regional idioms and seem to have no one native tongue" (17), mientras que Apter retoma textos, como su cuento "El viaje de Álvaro Rousselot" o el episodio sobre Max Mirebalais en La literatura nazi en América, para discutir la relación entre traducción y autoría (Against World Literature 317-319). Si bien la pregunta por el concepto de la traducción en la obra de Bolaño, al que estos estudios aluden, se discutirá en el tercer y cuarto punto del presente artículo, es necesario, en primer lugar, tratar brevemente algunos de los numerosos estudios (provenientes básicamente del campo latinoamericanista) acerca de la traducción de su obra. El problema que comparten todos esos trabajos reside en el hecho de que el término traducción apunta en realidad a la recepción de sus obras traducidas, es decir que en ninguno de ellos se investiga la traducción como texto sino más bien las lecturas críticas y las reseñas de esas traducciones que como tales se hacen "invisibles". Así, para dar algunos ejemplos, el capítulo de Bielsa dedicado a "Bolaño in translation” en su estudio sobre cosmopolitismo y traducción se limita, básicamente, a una labor recopilatoria de las reseñas en torno a la obra del autor en el ámbito hispanófono y anglófono (90-98). De manera parecida, el temprano y conocido artículo de Pollack ofrece una lectura crítica de los imaginarios estadounidenses en juego en su recepción y mercantilización, mientras que el estudio de Birns enfoca (de manera muy informativa, por cierto) la vida editorial de los textos del autor en Estados Unidos y sus diferentes traductores, pero sin referirse a las traducciones como tales. Otro ejemplo para el fenómeno de la práctica del "elogio no-fundamentado" de las traducciones de Bolaño ofrece Corral en su libro Bolaño traducido ${ }^{10}$ cuando habla, con respecto a Chris Andrews como uno de los tres traductores de Bolaño al inglés, de "las generalmente excelentes traducciones del australiano Andrews"

8 Consultar, por ejemplo, los artículos en Birns/De Castro o mis propias observaciones al respecto en Loy, "Deseos de mundo" y "Mocking World Literature".

9 Véase mi propia interpretación acerca de ese relato en Loy, "Deseos de mundo" y "Fantasmas". 10 Para una crítica más extensa a ese estudio, véanse Echevarría, "Bolaño internacional” y Loy, "Chistes”. 
(139) sin dar alguna explicación de esa presunta excelencia. De la misma manera, no precisa a qué se refiere al sostener que "[e]stá bien que los cuentos de Bolaño sean leídos como si hubieran sido escritos en inglés en vez de español" (140) y prefiere deshacerse de la tarea de una interpretación efectiva de las traducciones con el polémico comentario de que la pregunta por su calidad en realidad solo "ocasionará que algún nacionalista o provinciano latinoamericano quiera reclamar que algo se ha perdido en la presunta apropiación, como si la traducción fuera más que un arma funcional que les permite a los monolingües ampliar sus lecturas” (140). Esta posición parece tanto más equivocada si se tiene en cuenta que la obra de Bolaño por sus características formales -desde la coexistencia o mezcla de las múltiples variedades del español hasta la infinidad de alusiones (trans-)culturales e intertextuales- representa un sinfín de desafíos para cualquier traducción que merecen mayor escrutinio.

El acto de Walkowitz de incluir a Bolaño en su corpus de "born-translated literature" solo se justifica parcialmente, ya que, por un lado, su concepto abarca novelas en las que "translation functions as a thematic, structural, conceptual, and sometimes even typographical device" (4), cosa que, sin lugar a dudas, aplica para los textos del autor chileno; pero, por otra parte, parece difícil sostener con respecto a los libros de Bolaño que estos cumplan también con la segunda (y más prominente) parte de la definición de Walkowitz, es decir de pertenecer a "works [that] are written for translation, in the hope of being translated" (4). Si bien los debates por la "legibilidad" de la obra de Bolaño han sido numerosos ${ }^{11}$, vale señalar que factores como su tardío reconocimiento incluso en español y su temprana muerte difícilmente le hubieran permitido concebir sus obras desde un inicio en función de una futura traducción, o como lo ha formulado Ignacio Sánchez Prado: "Bolaño se destaca por el hecho de que su estética no parece haber cambiado de manera particularmente drástica como resultado del mercado. El trabajo altamente idiosincrático del estilo y su fuerte adherencia a juegos metaficcionales difícilmente podrían considerarse escritura mercantilizada" (29). Esta observación también rige especialmente para el lenguaje de Bolaño y los desafíos en relación a la ya nombrada copresencia múltiple de las variedades del español en su obra un punto que representaba, como han reconocido también sus traductores ${ }^{12}$, una

11 Véanse las observaciones al respecto en Altenberg (218).

12 Claro ejemplo de esto representa el comentario de Natasha Wimmer, quien tradujo al inglés, entre otros, Los detectives salvajes y 2666: "Yes, it was difficult. Idiomatic language is always one of the translator's biggest challenges. Bolaño draws on slang spanning continents and decades, from Mexico in the 1970s to Spain in the 1990s" (Esposito). 
dificultad particular al abordar la traducción de sus obras. Esta "glotopolítica"13 de Bolaño, como se podría llamar también, ha sido considerada por Hoyos como uno de los motivos fundamentales de su éxito mundial, al afirmar que "el escritor chileno se hizo global porque ofrece una escritura de la globalidad" (95). En la mezcla de variedades del habla, como es característica en la obra de Bolaño, Hoyos ve "una exploración de las condiciones de base de la experiencia transnacional, empezando por su registro oral" y no duda de la plena traducibilidad de estas formas de una enunciación híbrida al señalar que "Natasha Wimmer pudo capturar de esa rica textualidad en su traducción inglesa, bastó para causar un efecto potente, y lo mismo puede decirse de las traducciones a las otras lenguas que presiden sobre lo que Pascale Casanova llama république mondiale des lettres: el francés y el alemán” (95). Aparte de sumarse a la mencionada lista de los “elogios no-fundamentados” de las traducciones de Bolaño, la explicación de Hoyos no es convincente porque precisamente la copresencia de las distintas variedades del español, que en su versión original sí crean un efecto inmediato de un espacio narrativo "global" o "transnacional", se pierde irremediablemente en las versiones en inglés y, sobre todo, en francés y en alemán, ya que estos idiomas no disponen de un sistema lingüístico diatópico comparable al castellano. Con esto, la observación equivocada de Hoyos deja en evidencia la necesidad de una mayor atención que una crítica de la traducción de la(s) literatura(s) mundial (es) debería ostentar en sus juicios sobre la calidad y los aspectos de (in-)traducibilidad de este tipo de textos en el sentido de un balance más exacto de las ganancias y pérdidas translaticias ${ }^{14}$. A través de ejemplos puntuales de la novela Estrella distante (1996), a continuación se pretende dar cuenta de semejantes procesos al comparar exactamente las tres traducciones en los idiomas ya mencionados: inglés, francés y alemán.

13 Véase acerca de este concepto el libro de Marcellesi y Guespin, así como, para el contexto del castellano, sobre todo los estudios realizados por José del Valle. Si la noción glotopolítica del lenguaje se centra, como del Valle sostiene, en una "concepción fundamentalmente contextual del lenguaje [. . . ] donde se sitúan, por ejemplo, las distintas encarnaciones de la sociolingüística, que lo conciben como hecho social” (14), entonces no caben dudas de que la obra de Bolaño -y Estrella distante en especial- se inscriben en ese paradigma al considerar el lenguaje como un fenómeno siempre cambiante y dependiente de sus contextos sociales, temporales, geográficos y políticos frente a una noción autoritaria del lenguaje (en ese aspecto reside el motivo clave de la novela de relacionar el lenguaje autoritario de la dictadura de Pinochet con el discurso salvatorio de las vanguardias encarnado por el personaje de Carlos Wieder (véanse al respecto también mis comentarios en Loy, "La ironía”).

14 Locane formula una crítica parecida al concepto de Walkowitz y su interpretación superficial de Bolaño (142-149). 


\section{Las glotopolíticas literarias de Bolaño: tres traducciones de Estrella distante}

Si el aspecto de la traducción puede reclamar una importancia general para la escritura de Roberto Bolaño, es en su novela corta Estrella distante que quizás el lector se enfrenta de manera más intensa con una especie de "meta-novela translaticia”. Sin querer analizar aquí en profundidad la novela ${ }^{15}$, cabe señalar que la historia en torno de la búsqueda del poeta y asesino Carlos Wieder por un narrador alter-ego de Bolaño remite de manera constante y en el sentido más amplio a fenómenos de traducción: así, por ejemplo, aparece una serie de personajes enfrascados en proyectos de traducción, como un profesor chileno llamado Diego Soto sobre el que se dice:

También intentó traducir a Sophie Podolski, la joven poeta belga suicidada a los veintiún años (no pudo), a Pierre Guyotat, el autor de Eden, Eden, Eden y Prostitution (tampoco pudo), y La Disparition, de Georges Perec, novela policíaca escrita sin la letra $e$ y que Soto intentó (y sólo consiguió a medias) trasladar al español aplicándose en lo que Jardiel Poncela había hecho medio siglo antes en un relato en donde la consabida vocal brillaba por su ausencia. Pero una cosa era escribir sin la e y otra muy distinta traducir sin la e. (76)

Ya a partir de este ejemplo se podría desarrollar una serie de reflexiones sobre la importancia de la traducción en la novela: por un lado, destaca la idea de la traducción como esa forma de acceder a otros "textos del mundo"16, que implica siempre preguntas de (in-)visibilidad y del canon, como lo demuestra aquí el comentario irónico acerca del precursor español (prácticamente olvidado) de Perec; por el otro lado, se hace énfasis en una separación de los actos de escribir y de traducir, así como en el hecho de que las traducciones pueden perfectamente fracasar. Cabe destacar, sin embargo, que, más allá de esta presencia explícita de actos translaticios en la novela y los temas de la memoria o de la herencia de las vanguardias, que han dominado las lecturas críticas de la novela, su núcleo, en un sentido más amplio, consiste en una reflexión sobre un mundo dominado por una genuina falta de estabilidad de los signos y, por ende, del lenguaje mismo. Este fenómeno afecta, en primer lugar, la constitución del relato que se presenta, en el prólogo muchas veces citado, como una reescritura del último capítulo de $L a$

15 Para diferentes lecturas de la novela, véanse, por ejemplo, mis propuestas en Loy, "Escritores bárbaros", "De oposiciones" y "The Precarious".

16 Hay que tener en cuenta que una de las primeras apariciones documentadas de Bolaño como autor se efectúa como autor de traducciones de una "nueva poesía francesa" en la revista Plural en 1977. Acerca de la intertextualidad en la novela (y en especial de la antipoesía de Nicanor Parra), véase mi análisis en Loy, “Chistes”. 
literatura nazi en América en forma de "una historia más larga, no espejo ni explosión de otras historias sino espejo y explosión en sí misma” (11). La novela se exhibe como un texto concebido de manera dialógica -entre Bolaño, Belano y "el fantasma cada día más vivo de Pierre Menard" (11) - que rechaza -como subraya la referencia a Borges- la idea del "original" ${ }^{17}$ y la posibilidad de una estabilidad y de un control de los signos y de los significados. En ese sentido, el texto ostenta en todos sus niveles exhibe su carácter "translaticio": la reescritura le confiere un estado de "derivado", todas las identidades de los personajes -empezando por la del criminal buscado- se tornan inciertas porque siempre se basan en testimonios, historias y otras huellas materiales "ambiguas" (como la serie de fotografías presentadas como inciertas y borrosas), que en su conjunto componen un universo sígnico radicalmente inestable ${ }^{18}$. Si Antoine Berman en sus trabajos traductológicos postula que una de las bases metodológicas dentro de la crítica de traducción sea "un patient travail de sélection d'exemples stylistiques (au sens large) pertinents et significatifs dans l'original [...], les zones signifiantes où une œuvre atteint sa propre visée [...] et son propre centre de gravité” (70), entonces el argumento aquí sería que en Estrella distante son precisamente las zonas en las que la inestabilidad del lenguaje como tal se hace más evidente las que habría que investigar con preferencia y desde una perspectiva comparada ${ }^{19}$.

17 Walkowitz subsume también este fenómeno bajo sus "born-translated novels" al advertir que frente a muchos de esos textos "[r]eaders are asked to experience the text as a delayed or detoured object: a book that began somewhere else” (30).

18 Este hecho también conlleva una dimensión ética del texto que se hace visible, para nombrar solamente un ejemplo específico, en el asesinato de Carlos Wieder por parte del detective chileno Abel Romero al final de la novela: no queda claro si Wieder realmente es el autor de todos los crímenes que bajo distintos nombres ha cometido porque Romero en sus investigaciones se basa en una serie de testimonios dudosos como el de Joanna Silvestri, una exactriz porno cuyas declaraciones sobre el caso no aparecen en la novela sino en un cuento de la colección Llamadas telefónicas. El lector de la novela solamente dispone de una "traducción" de esta conversación de Romero y Silvestri que, además, se realiza entre un chileno y una italiana en francés y luego es relatada en castellano a Belano por Romero y, finalmente, resumida por el narrador para el lector real de la novela - una serie que escenifica, otra vez más, el principio fundamental de la cadena de relatos y la inseguridad del significado "real" o "auténtico" de un hecho deformado por la comunicación misma.

19 Véase también el argumento de Berman acerca de la comparación de traducciones: "Les 'solutions' apportées par chaque traducteur à la traduction d'une œuvre [.. .] sont si variées, si inattendues, qu'elles nous introduisent [...] à une double dimension plurielle: celle de la traduction, qui est toujours les traductions [...]. Le lecteur ou l'auditeur [..] est ainsi amené à se libérer de toute naïveté et de tout dogmatisme” (85). 
No resulta difícil reconocer que todas las traducciones de Bolaño generalmente cumplen con la primera pregunta que, según Berman, una crítica de la traducción tiene que aclarar: si la traducción "funciona" ("si le texte traduit 'tient”” (65)). En el caso de los tres idiomas en cuestión acá -inglés, francés y alemán- cabe destacar que Bolaño ha contado con un total de diez traductores diferentes: Chris Andrews y Natasha Wimmer en el caso de los textos narrativos y Laura Healy de la poesía en inglés, Robert Amutio para la obra completa en francés con excepción de Amuleto (traducida por Emile y Nicole Martel para la editorial canadiense Les Allusifs) y Heinrich von Berenberg y Christian Hansen en alemán. Sin indagar profundamente en las biografías profesionales de todos los traductores ${ }^{20}$, se recurrirá a lo largo del análisis a determinados comentarios en relación a sus respectivas percepciones de la obra de Bolaño en traducción.

Si el desafío particular de traducir las obras de Bolaño consiste, como se acaba de mencionar, en la presencia simultánea de las múltiples variedades del español y si el núcleo de Estrella distante representa la inestabilidad de los signos y del lenguaje, entonces resulta lógico enfocar el siguiente análisis en ejemplos que ilustren precisamente el manejo de estos dos aspectos por parte de los traductores de la novela, quienes son Andrews, Amutio y Hansen. Un punto decisivo en cuanto a la forma lingüística de la narración es la alternancia de la variedad chilena y otra "peninsular"/"ibérica” del español, que en el relato biográfico del narrador se superponen continuamente y vuelven borrosas las fronteras espacio-temporales de la historia: esto se hace evidente, por ejemplo, cuando el narrador (chileno) relata un encuentro en los días posteriores al Golpe de Estado de 1973 con dos amigas-poetas de su taller en el sur de Chile con las siguientes palabras: "Me dijeron que se iban, pero no al extranjero sino a Nacimiento, un pueblo a pocos kilómetros de Concepción, a la casa de sus padres. Qué alivio, dije, pensé que os marchabais a Suecia o algo asî” (27). Destaca el hecho de que el narrador usa aquí la segunda persona del plural propia del español peninsular y ausente en la variedad chilena, por lo que resulta altamente inverosímil que el comentario del narrador en su momento se haya hecho literalmente de esta manera. Más bien, la irrupción de la variedad peninsular señala aquí ya al inicio del relato el futuro destino del exilio y el traspaso a España - un indicio dentro

20 Se podría destacar el hecho de que Wimmer, Hansen y Amutio son sobre todo traductores (habiendo Amutio contribuido también de manera decisiva a que se adquirieran los derechos en francés por parte de Christian Bourgois) mientras que el “descubridor” alemán de Bolaño, von Berenberg, ha dedicado su carrera profesional en primer lugar a la edición. Andrews, en cambio, no es solamente traductor sino también poeta y profesor universitario así como autor de una de las obras críticas más logradas que hasta el momento se han publicado sobre Bolaño. 
de la estructura detectivesca de la novela que en las traducciones necesariamente tiene que desaparecer ${ }^{21}$. Mientras que en este ejemplo es fácil de "esconder" ese aspecto en la traducción, hay otro caso que remite a la misma problemática de la copresencia de variedades lingüísticas pero con la diferencia de que se hacen explícitos el traslado del narrador de Chile a España y las consecuencias para su propio uso del lenguaje: "Nosotros casi nunca teníamos plata (es divertido escribir ahora la palabra plata: brilla como un ojo en la noche)" (16). La clave de esta frase es la siguiente: la reacción afectiva que formula el narrador -“es divertido escribir ahora la palabra plata" - surge a raíz de esta diferencia espacio-temporal-lingüística que separa su pasado chileno y el presente español (o más bien: catalán) desde el que escribe. Dicho de otra manera: el uso de la palabra "plata" como expresión corriente (coloquial) de la variedad chilena del español le llama la atención precisamente porque en su entorno ibérico debe haber dejado de usarla, siendo "pasta" el término coloquial correspondiente de la variedad peninsular. El problema para la traducción resulta del hecho que tiene que encontrar una solución para hacer plausible la reacción de asombro del narrador ante una diferencia lingüística que no se puede traducir literalmente a los tres idiomas en cuestión porque desconocen prácticamente esta simultaneidad de variedades diatópicas. En la versión inglesa, Andrews escribe lo siguiente: "We hardly ever had two dimes to rub together (it seems so odd to be writing the word dime. I can see it shining like an eye in the night)" (6). Andrews apela a una expresión idiomática más allá del lexema singular del original que es llamativa porque recurre, en el uso de la palabra "dime”, a una opción que conlleva una marca geográfica clara del inglés estadounidense ${ }^{22}$. Las implicaciones de esta decisión resultan interesantes: si el narrador de Andrews afirma que le parece “odd” el uso de la palabra “dime”, entonces el lector en inglés aquí debe asociar ese asombro del narrador (de igual manera que en su original en español) con el hecho de que el uso del inglés estadounidense para él ha perdido una familiaridad que alguna vez probablemente había tenido para él. Andrews, por lo tanto, opta por la opción "geográfica” porque el inglés es capaz de ofrecerla de manera parecida al español. Esta hipótesis se refuerza probablemente al considerar que la

21 Véanse la versión inglesa ("What a relief, I said, I thought you were going to Sweden or somewhere like that" (17)) y francesa ("Je suis content, dis-je soulagé, je croyais que vous partiez pour la Suède ou un pays de ce genre” (29)). Hansen, en la traducción alemana, comete un error llamativo al confundir Suecia con Suiza cuando escribe: "Na, ein Glück, sagte ich, ich dachte, ihr würdet in die Schweiz gehen oder sonstwohin” (24-25). Aplica para este caso probablemente el comentario lacónico de Berman de que hasta los mejores traductores de repente pueden caer en un "profundo sueño".

22 Véanse, para comprobar esta hipótesis, por ejemplo, los datos históricos acerca del uso de la palabra en los corpus del Corpus of Historical American English y del British National Corpus. 
primera traducción en inglés para New Directions fue publicada efectivamente en Inglaterra $^{23}$, por lo que la presencia de un giro "americano" aquí claramente podría causarle un efecto parecido a un lector inglés en el sentido de percibir el asombro del narrador a raíz de una diferencia de variedades del lenguaje. Si bien Andrews en una entrevista ha señalado lo problemático que resulta el uso de regionalismos en inglés ${ }^{24}$ y la necesidad “[to] rely on other markers of locality in the context” (Wilson), aquí pareciera haber optado igualmente por una variante que en inglés funciona bien, ya que hace plausible la reacción del narrador (aunque resulta más bien curioso si se sigue desarrollando la idea porque, si la variedad estadounidense le resulta extraña al "Bolaño de Andrews", entonces la conclusión tendría que ser que el traslado en la traducción en realidad no fue entre Chile y España sino entre Estados Unidos e Inglaterra o Irlanda o Australia) ${ }^{25}$.

En la versión alemana, en cambio, Christian Hansen opta por la siguiente traducción: "Wir waren fast immer blank (lustig, heute das Wort blank zu benutzen; es leuchtet wie ein Auge in der Nacht)" (13). Hansen sustituye la palabra "plata" por el adjetivo "blank" que forma parte de otro giro idiomático: "blank sein” en alemán significa "no tener dinero", de manera idéntica a la expresión “estar sin blanca” en español. En alemán, el traductor no tiene la posibilidad de recurrir a una marca geográfica, ya que -de una forma mucho más extrema que

23 Véase el comentario de Andrews al respecto: "When the first couple of English translations, By Night in Chile and Distant Star, came out in England, they weren’t published in America straight away" (Heyward). Asimismo, Andrews resalta este aspecto del lugar de la publicación y su uso del lenguaje: "It depends where the commissioning is done, and what's happened with Bolaño has been a little strange because the commissioning shifted from the UK to the US after the first three books. When it has been for an English publisher, I have written, for example, "flat" rather than "apartment." I've never really used many "Australianisms" though. I've tried to smuggle a few in here and there, but it's tricky, and it's hard to get away with! It would be different if you were working for an Australian publisher, publishing for an Australian market. It has occasionally been tempting to use an "Australianism" to translate an expression from Chilean Spanish, but the risk is that it would be merely confusing, even for an Australian reader" (Heyward).

24 "One difficulty that crops up frequently in Bolaño is how to translate regional familiar language: Mexican or Chilean slang, for example. If you use regional terms in English it can be confusing for the reader, because they will hear the Chilean or Mexican character as an Australian, say" (Wilson).

25 Veáse también el comentario de Venuti al respecto de este fenómeno: “[t]he localizing drive of translation can [...] change the very nature of the categories 'foreign' and 'local' as they are understood by readerships in the receiving situation" ("World Literature" 182). Aparte de la dimensión geográfica, se podría argumentar que Andrews también logra incorporar una dimensión temporal ya que los corpus señalan que el uso de ese giro ha ido disminuyendo levemente en las últimas tres décadas, cosa que le confiere más plausibilidad al hecho de que entre el momento de la afirmación del narrador a mediados de los años 90 y su pasado en Chile en los 70 existe igualmente una diferencia de tiempo considerable. 
en inglés- las únicas posibilidades serían el uso de uno de los dialectos regionales alemanes o de un término del alemán suizo o austríaco, todas opciones que tendrían un efecto grotesco porque transformarían al narrador chileno en España fácilmente en un bávaro o berlinés perdido en la Costa Brava ${ }^{26}$. No obstante, el uso de la expresión "blank sein” hace plausible la reacción del narrador también en la traducción porque posee en alemán un carácter algo anticuado ${ }^{27}$ que es capaz de sugerirle al lector alemán una diferencia temporal (en el sentido de una expresión que el narrador usaba en su juventud probablemente) como causa de $\mathrm{su}$ asombro ${ }^{28}$. En la versión francesa, finalmente, Robert Amutio escribe: "Nous n'avions presque jamais d'argent (c'est drôle d'écrire maintenant le mot argent: il luit comme un œil dans la nuit)" (17). A diferencia de Andrews y Hansen, Amutio traduce la frase del original en su sentido literal $-\mathrm{y}$ fracasa con esto porque no logra hacer plausible la reacción del narrador-: "argent” no transporta ningún significado que pudiera justificar su asombro (o más bien: el divertimiento del narrador porque, al igual que Hansen, opta por la traducción en el sentido de “cómico" en vez de "bizarre” o “curieux" que sería probablemente más adecuado). En la simple repetición del lexema no se logra crear ninguna tensión espacio-temporal, el efecto aquí se pierde y hasta queda, para el lector francés, sin sentido (¿por qué debería ser divertido volver a repetir esa palabra? Probablemente también en este caso una solución idiomática como "Nous n’avions presque jamais de sous" hubiera sido preferible $)^{29}$.

26 El efecto inverso y practicado a lo largo de toda la obra de Bolaño se puede observar, por ejemplo, en el caso de su novela póstuma El Tercer Reich en la que un empleado alemán de Stuttgart pasa sus vacaciones en la Costa Brava con su novia y entra en contacto tanto con los locales como con otros turistas alemanes y donde todos conversan, por supuesto, en castellano y sin problemas aparentes de comunicación. El mismo efecto se reproduce en 2666, sobre todo en la parte de los críticos (véase al respecto también el trabajo de Altenberg).

27 Véase también en el diccionario Digitales Wörterbuch der deutschen Sprache que constata una clara caída en desuso después de los años 60.

28 Otro punto interesante al comprar las opciones de Andrews y Hansen es que la palabra "divertido", que en el español chileno se usa más bien en el sentido de "raro", es traducida por Andrews muy adecuadamente con "odd" mientras que Hansen opta por "lustig” que borra la ambigüedad de "divertido" porque en alemán significa solamente "gracioso"/"chistoso" (en cambio, una palabra como "komisch" podría haber conservado ese vaivén entre "raro" y "gracioso" como uno de los principios operantes de la novela con sus pasajes humorísticos y bizarros).

29 Le agradezco al poeta franco-chileno Carles Díaz las intensas discusiones sobre este punto. 
Otros casos que demuestran bien las diferentes estrategias de traducción se refieren, por ejemplo, al grado divergente de los textos con el que exhiben elementos "extraños" del original o aspiran a crear esa "ilusión de transparencia" tan criticada por Venuti. En Estrella distante se encuentran varias menciones en ese sentido. Así, como muestra de lo anterior, dice el narrador en un momento sobre su experiencia en los días después del Golpe de Estado: "Pero yo tenía ganas de cantar y de bailar y las malas noticias (o las elucubraciones sobre malas noticias) sólo contribuían a echarle más leña al fuego de mi alegría, si se me permite la expresión, cursi a más no poder (siútica hubiéramos dicho entonces)" (27-28). Mientras que Andrews opta por una traducción que esta vez usa una marca temporal para expresar la diferencia geográfica del original (siútico es una palabra marcada sobre todo por el registro chileno y no por un desuso en el tiempo $)^{30}$ y la versión de Hansen fracasa en ese ejemplo ${ }^{31}$, Amutio opta simplemente para mantener el término al escribir: "si on me passe l'expression, d'un mauvais goût absolu (nous aurions dit siútica dans l'argot d'alors)" (31). Igualmente interesante en ese sentido es otra frase de la novela en la que el narrador se refiere a un poema llamado "Saranguaco" de Nicanor Parra para burlarse de las incoherencias de la poesía de Carlos Wieder con las palabras: "(Más exactamente: con un inicio que no hubiera desaprobado Isidore Isou y con un final inédito digno de un saranguaco)” (42). Mientras Hansen mantiene la opacidad ${ }^{32}$ (que, sin lugar a dudas, también para un lector común y corriente en español debe resultar oscura si no conoce el texto de Parra) y Amutio hace explícita la referencia intertextual ("et une fin inédite digne du Saranguaco de Nicanor Parra” (48)), Andrews opta por una simplificación radical al poner "(while the unexpected ending would not have been out of place in a Chilean folk song)" (31-32). A pesar de que Andrews en otro momento reconoce que "[j]ust occasionally, I think, the best solution is to leave the word in Spanish, but only very occasionally" (Wilson), se puede observar

30 Andrews escribe: "to use an expression which is, I admit, impossibly trite ('corny' we would have said back then)” (18). "Corny”, según los corpus del inglés, aparece más o menos de manera igual en las variedades de Estados Unidos y de Inglaterra, pero ostenta una leve caída en desuso en las últimas tres décadas.

31 La versión alemana pone: "wenn ich mir diese abgeschmackte Formulierung erlauben darf ("kitschig' hätten wir damals gesagt)" (26). Aquí no hay ninguna lógica de marcar la palabra "kitschig" como presuntamente "pasada de moda" sino que incluso habría que cambiar ambos términos de posición porque "kitschig" experimenta, según las estadísticas del DWDS, un fuerte auge a partir de los años 80 mientras que "abgeschmackt” pierde claramente importancia a partir de los 90 .

32 "(genauer: mit einem Anfang, dem Isidore Isou seine Billigung nicht versagt, und einem unerhörten Schluß, der einem saranguaco gut zu Gesicht gestanden hätte)” (41). 
en su traducción una cierta tendencia a "limar" el texto con respecto a la presencia de palabras españolas y reducir, de esta manera, el grado de "extrañeza” para el lector del texto traducido -un hecho que encuentra probablemente su correspondencia en el diseño de las portadas en la versión inglesa/estadounidense y francesa-: mientras que las primeras optan por una "plasticidad" al enmarcar el título en un paisaje de los Andes con un avión cruzando el cielo y remitiendo básicamente al "plot" del relato con un cierto toque exotizante, la portada francesa muestra un fragmento de la pintura Coigitum, del artista chileno Roberto Matta, cuya biografía y obra permiten en muchos sentidos una relación mucho más compleja frente al relato de Bolaño ${ }^{33}$.

Dos conclusiones pueden extraerse de estas observaciones: la primera es que los textos de Bolaño poseen múltiples dimensiones lingüísticas que se resisten a una traducción sencilla (en el sentido de ser "born-translated novels" concebidas para la traducción) y que las soluciones propuestas por sus traductores en la mayoría de los casos se pueden considerar logradas, y en otros no tanto; la segunda conclusión -paradójica y lógica a la vez- es que estas microresistencias, como las que se han analizado aquí, en los libros de Bolaño influyen poco o nada en la percepción general de sus novelas en traducción y menos aún en su éxito mundial. Este último punto requiere una explicación para el que los comentarios de los traductores de Bolaño bien pueden servir porque confirman de alguna manera esta hipótesis: las obras de Bolaño se resisten parcialmente a la traducción, pero la mayoría -y las más importantes y características- de sus rasgos distintivos, sobre todo sintácticos, se conservan también al traspasar sus textos a otros idiomas. En ese sentido hay que entender el comentario de Chris Andrews cuando afirma en otra entrevista que:

The first thing I should say is that there are always losses and shifts in translation, and Bolaño is no exception. But I do think that his style comes through relatively strongly, whether it's me translating him or someone else. When I read Natasha Wimmer's translations of his other books, I feel like I'm straight back into the world of Bolaño, and I don't feel any resistance or drag or loss. That is partly because some of the key stylistic features in his work are quite large-scale, like the head-long sentence that keeps rolling on via coordination, for example, with, and, and and, and and. (Heyward)

33 La versión alemana simplemente muestra el nombre del autor y el título de la novela en una tapa negra al lado de una representación estilizada del apellido de Bolaño quien, a la fecha de la publicación de esta versión de la traducción en 2010, ya podía funcionar como esa "marca” capaz de atraer a los lectores únicamente a través de su nombre. 
Esto significa que -a pesar de los problemas que el fenómeno de las múltiples variedades diatópicas del español le puede causar a las traducciones en no pocas ocasiones- el rasgo reconocible de la prosa bolañesca, que también se puede conservar sin mayores problemas en otros idiomas, son el ritmo y las estructuras sintácticas de su estilo. De manera muy parecida, Heinrich von Berenberg ha destacado este aspecto al hablar en una entrevista de la "musicalidad" de sus narraciones que, para el traductor de Los detectives salvajes y La literatura nazi al alemán, son "el elemento formal determinante” en Bolaño (Kleinemeier). También Christian Hansen destaca su decepción inicial con la prosa de Bolaño que sentía, en cuanto a su expresividad, como "funcional, de Bauhaus" hasta descubrir que el rasgo distintivo y cautivador se revelaba a nivel estructural de los textos. Hansen habla también del efecto de un "folioscopio" (Mortati 54) y destaca que "[e]n el caso de Bolaño tienes que crear esas configuraciones, esas atmósferas. Hay ciertos cuentos de él que leo con lágrimas en los ojos y si ese efecto no se produce también en la traducción, no me parecería lograda. No se trata de una palabra, ni de una frase, sino que es el conjunto" (Mortati 55). Asimismo, Robert Amutio sostiene:

Chez Bolaño, il y a aussi bien sûr des éléments plus ou moins facilement traduisibles [...]. J'ai rencontré il y a quelques jours les traducteurs vers l'anglais et vers l'allemand de Bolaño, et si je les crois, qu'on lise Bolaño dans une langue ou dans une autre, on reconnaît le « style » bolanien [...]. Ça ne signifie pas évidemment que Bolaño soit un auteur facile à traduire! (Anquetil)

El estilo y el lenguaje figurativo ${ }^{34}$ representan, entonces, mucho más que las dimensiones lingüísticas de su español "híbrido" que se han analizado aquí, los factores decisivos de la traducibilidad de Bolaño. En ese sentido, bien se podría denominar sus obras -tal como lo ha hecho Andrews en repetidas ocasiones- como "quite robust, which is not to say that they're easy to translate" (Wilson). Esta "robusteza" de la obra, que se refiere sobre todo a su capacidad de proteger su "carácter” y sus rasgos distintivos en la traducción, corresponde a la idea de la "obra elástica”, como la he formulado en otro contexto ${ }^{35}$, como un motivo decisivo del éxito mundial de Bolaño: si el autor chileno se caracteriza, en palabras de Echevarría, por una "infrecuente capacidad de conectar con lectores de todo tipo" (176), entonces parece radicar ahí un punto clave de su recepción global y, muy importante también, de su mercantilización radical de los últimos diez años: como en pocos autores, la obra de

34 "Many of the figures and the kind of post-surrealist images that come in strange bursts in his prose are also pretty amenable to translation", afirma Andrews (Heyward).

35 Véase mi artículo sobre la recepción alemana de Bolaño en Loy "Der Teil der Kritiker”. 
Bolaño es capaz de satisfacer tanto las inquietudes de un lector joven y naif, que se identifica con los plots de aventura y viajes en Los detectives salvajes, como las del filólogo versado en las lecturas del canon que saca un placer inmenso al descifrar los crucigramas intertextuales ${ }^{36}$ que caracterizan toda su obra. Es esta posibilidad de ofrecer al mismo tiempo lecturas hípercomplejas y reducciones más bien extremas, de funcionar tanto en el gran canon literario como desde la perspectiva de los lectores aficionados de las buenas historias, la que transformó a Bolaño -junto con su biografía perfectamente "vendible" desde las perspectivas del mercado angloamericano- en un candidato ideal para transformarse en un autor mundial del siglo XXI. Ante ese panorama es importante realzar el hecho de que la glotopolítica de Bolaño refleja esa dialéctica entre resistencia y traducibilidad que abre su obra hacia múltiples lecturas: por un lado, la "variedad de variedades" lingüísticas con sus potenciales resistentes es, como se ha visto, capaz de mantener un grado de "extrañeza" en las traducciones (a diferencia de esas "born-translated novels" limadas desde un inicio para su circulación internacional sin dificultades), por lo que Bolaño se diferencia de lo que Echevarría ha denominado como "estilo internacional” que acompaña las generaciones jóvenes de autores latinoamericanos en su afán de integrarse a un mercado literario global que "incentiva entre los jóvenes escritores una estandarización de la lengua y una estereotipificación de los planteamientos narrativos" ("Lengua", 186). En ese sentido, el lenguaje literario de Bolaño se resiste a una plena recuperación en traducción sin ser por eso "untranslatable”, es más, en la persistencia de ciertos elementos lingüísticos difíciles o imposibles de traducir estos creen lo que Andrews ha llamado "a strong flavor" (Heyward), por lo que se podría decir que es precisamente ese grado limitado de "otredad" lingüística que distingue a Bolaño de buena parte de una prosa "funcional” y le confiere una singularidad que, por otro lado, hace que su capacidad de circulación más bien aumente en vez de disminuir ${ }^{37}$. Lo particular de estos aspectos y de la recepción global de

36 En mi reciente libro (Roberto Bolaños wilde Bibliothek) ofrezco una serie de interpretaciones de esas dimensiones intertextuales de su obra completa en relación a autores tan diversos como Georges Perec y Luis de Góngora, Enrique Lihn y Ernst Jünger, Sor Juana Inés de la Cruz y Charles Baudelaire.

37 Es interesante observar como esta glotopolítica de Bolaño en el sentido de una posición relativa entre resistencia y accesibilidad frente al mercado literario corresponde en muchos sentidos a sus políticas de autoría y su estética en general; véase acerca de ese argumento el artículo de Cobas Carral y Garibotto. Que ese tipo de glotopolíticas es un factor importante sobre todo para la literatura latinoamericana se explica ante el hecho de la dominación española internacional del mercado editorial en castellano (véase, acerca de este aspecto, por ejemplo, el estudio de Yúdice). Sin poder indagar aquí en este aspecto, baste con un testimonio muy ilustrativo que da el autor colombiano Evelio Rosero sobre su novela Juliana los mira 
Bolaño es que -sin querer caer aquí en el género de lo profético- refleja, con una exactitud algo inquietante, precisamente la propia poética (translaticia) como la formuló el autor en diferentes escritos y que se analizará brevemente en la parte final del presente estudio.

\section{4 "La traducción es un yunque": la (in-)traducibilidad en Bolaño}

La traducción y sus frecuentes fracasos en un mundo globalizado ${ }^{38}$ son un tema recurrente en Bolaño. Sin embargo, han sido relativamente escasos los comentarios explícitos del autor sobre el tema, con la importante excepción de un breve texto ensayístico titulado "La traducción es un yunque" e incluido en el volumen Entre paréntesis. En este, Bolaño diferencia en la literatura mundial, en un primer paso, entre "una obra maestra absoluta de una obra maestra a secas, o, si es posible decirlo, una literatura viva, una literatura patrimonio de todos los hombres, de una literatura que sólo es patrimonio de determinada tribu o de un segmento de determinada tribu" (223). Mientras que Cervantes forma parte de la primera categoría, autores como Quevedo y Góngora -debido a su complejidad lingüística prácticamente intraducible de forma "adecuada" a otros idiomas- representan la segunda. El punto clave de las reflexiones de Bolaño consiste en proponer la

y su destino luego de haber sido finalista del Premio Herralde en 1986: "Yo estaba muy contento por el premio Anagrama. Mi agente entonces era Carmen Balcells, una gran señora y una gran lectora que me guió mucho. El problema empezó cuando me llegaron las pruebas del libro para que yo le diera la última leída. Tenía cambios totales. Yo tengo diálogos sin puntuación y ellos los puntuaron. Luego hablé con ellos personalmente y los veía escandalizados: en lugar de escribir matera escribían maceta; yo escribía ‘debe ser que fulanito' y ellos ‘debe de ser’; ese 'debe de’ a mí me parecía cacofónico y se los dije. [.. .] Publicaron mi novela, pero nunca la trajeron a Colombia, nunca la enviaron a un periodista o a un crítico, se quedó encerrada durante diez años en una bodega. Fue una mala jugada por parte de estos editores" (Maldonado Tovar).

38 Sobre todo 2666 exhibe este aspecto en toda una serie de escenas de la novela como, por ejemplo, en el famoso episodio en los que los críticos literarios europeos agreden a un taxista pakistaní en Londres o cuando se describe el viaje de unos antropólogos franceses a Borneo y su contacto con un grupo de indígenas. Con respecto al primer episodio, véase también Altenberg; sobre el problema de la traducción en el contexto de la crisis del cosmopolitismo en 2666 también Loy, "Der Teil der Kritiker". Que sobre todo "La parte de Fate” en 2666 integra estas preguntas también a nivel del lenguaje mismo en forma de un español "mexicano" inventado por Bolaño ha demostrado el estupendo análisis de Levinson. 
traducción de una obra como el método decisivo para "probar" su calidad literaria, es decir como ese "yunque" en el que se revela bajo los golpes del "herrero traductor" el potencial de resistencia de un texto:

¿Cómo reconocer una obra de arte? [...] Es fácil. Hay que traducirla. Que el traductor no sea una lumbrera. Hay que arrancarle páginas al azar. Hay que dejarla tirada en un desván. Si después de todo esto aparece un joven y la lee, y tras leerla la hace suya, y le es fiel (o infiel, qué más da) y la reinterpreta y la acompaña en su viaje a los límites y ambos se enriquecen y el joven añade un gramo de valor a su valor natural, estamos ante algo, una máquina o un libro, capaz de hablar a todos los seres humanos [...]. (223-224)

El hecho de que Bolaño considere que una traducción fracasada -“que el traductor no sea una lumbrera”- es la mejor forma para reconocer si una obra literaria “sobrevive" en la traducción corresponde de manera exacta a los resultados de la breve comparación de las traducciones de su obra realizadas anteriormente: aunque estas -al igual que probablemente todas las traducciones de sus libros- no sean perfectas ni puedan transportar (a pesar de la calidad innegable de sus traductores) todos los matices del original ${ }^{39}$, la literatura de Bolaño es capaz de surtir esos efectos en sus lectores mundiales. El factor de la fidelidad - "fiel (o infiel, qué más da)" - no es de importancia para el texto traducido mientras que la traducción sepa trasladar lo que Bolaño llama, en relación al ejemplo del Quijote, su "razón" (222) que hace que "se imponga o impregne la imaginación de miles de lectores, a quienes no les importa ni el lujo verbal ni el ritmo ni la fuerza de la prosodia cervantina que obviamente cualquier traducción, por buena que sea, desdibuja o disuelve” (222-223). Las modalidades de este proceso, sin embargo, (y aquí nos encontramos en el punto límite de la crítica de la literatura mundial y sus aspiraciones a describir los funcionamientos de su objeto) tienen que sustraerse necesariamente a los intentos de verbalización: son, como lo describe Bolaño a partir del ejemplo de una representación infame de Macbeth visitada por Borges, un efecto que surge cuando el lector -más allá de los contextos de la obra entre las que también contarían la traducción y su calidad- "vuelve a sumergirse en el destino de aquellos seres que atraviesan el tiempo y vuelve a temblar con aquello que a falta de una palabra mejor llamaremos magia” (223).

39 Amutio relata la negación de Bolaño de intervenir en mayor grado en el proceso de traducción aunque sus conocimientos del francés se lo hubieran permitido sin problemas: "Il me laissait la liberté et la responsabilité de faire ce que je croyais être le mieux pour l'œuvre” (223). 


\section{Bibliografía}

Anquetil, Marilyn et al. “Entretien avec Robert Amutio, traducteur”. Littexpress, 2011. http:// littexpress.over-blog.net/article-entretien-avec-robert-amutio-traducteur-65470640.html, consultado 8 de enero 2019.

Altenberg, Tilmann. "Bolaño against Babel: Multilingualism, Translation and Narration in 2666, 'La parte de los críticos'”. Bulletin of Hispanic Studies, vol. 95, no. 2, pp. 217-233.

Amutio, Robert. "Paroles de traducteur”. Les astres noirs de Roberto Bolaño. Eds. Karim Benmiloud y Raphael Estève. Presses Universitaires de Bordeaux, 2007, pp. 219-228.

Andrews, Chris. Roberto Bolaño's Fiction: an Expanding Universe. Columbia University Press, 2014.

Apter, Emily. Against World Literature. On the Politics of Untranslatability. Verso, 2013.

--.. The Translation Zone. A New Comparative Literature. Princeton University Press, 2006.

Auerbach, Erich. Dante als Dichter der irdischen Welt. De Gruyter, 2001.

Berman, Antoine. Pour une critique des traductions: John Donne. Gallimard, 1995.

Bielsa, Esperança. Cosmopolitanism and Translation. Investigations into the experience of the foreign. Routledge, 2016.

Bolaño, Roberto. Stern in der Ferne. Trad. Christian Hansen. Fischer, 2010.

--.. Distant Star. Trad. Chris Andrews. Vintage, 2009.

--.. 2666. Anagrama, 2004.

--.. “La traducción es un yunque”. Entre paréntesis. Anagrama, 2004, pp. 222-224.

---. Étoile distante. Trad. Robert Amutio. Christian Bourgois, 2002.

--.. Estrella distante. Anagrama, 1996.

--.. “El universo hinchado. Nueva poesía francesa”. Plural, 64, 1977, pp. 20-24.

Birns, Nicholas. "The Part about the Critics: the World Reception of Roberto Bolaño". Critical Insights: Roberto Bolaño. Ed. Ignacio López-Calvo. Salem Press, 2015, pp. 50-64.

Birns, Nicholas y Juan De Castro (eds.). Roberto Bolaño as World Literature. Bloomsbury, 2017. Cobas Carral, Andrea y Verónica Garibotto. “Un epitafio en el desierto. Poesía y revolución en Los detectives salvajes”. Bolaño salvaje. Eds. Edmundo Paz Soldán y Gustavo Faverón. Candaya, 2008, pp. 163-189.

Corral, Wilfrido. Bolaño traducido: nueva literatura mundial. Escalera, 2011.

Damrosch, David. What is World Literature? Princeton University Press, 2003.

Echevarría, Ignacio. "Bolaño internacional: algunas reflexiones en torno al éxito internacional de Roberto Bolaño". Estudios Públicos, no. 130, 2013, pp. 175-202.

--.. "Lengua e identidad". Desvíos: un recorrida crítico por la reciente narrativa latinoamericana. Ediciones Universidad Diego Portales, 2007, pp. 185-188.

Esposito, Scott. “The Natasha Wimmer Interview". The Quarterly Conversation, 2008. http:// quarterlyconversation.com/the-natasha-wimmer-interview, consultado 8 de enero 2019.

Goethe, Johann Wolfgang. "German Romance”. Sämtliche Werke, Vol. 22. Eds. Friedmar Apel y Hendrik Birus. Suhrkamp, 1999, pp. 432-434.

Grossman, Edith. Why Translation Matters. Yale University Press, 2010.

Guespin, Louis y Jean-Baptiste Marcellesi. “Pour la glottopolitique”. Langages, no. 83, 1986, pp. 5-34.

Hansen, Christian. “Aus dem Übersetzernähkästchen: Flaulfleisch-Prosa”. Wilde Leser, 2009. http://www.wilde-leser.de/?p=469, consultado 8 de enero 2019. 
Harrison, Nicholas. "World literature: What gets lost in translation?" The Journal of Commonwealth Literature, Vol. 49, no. 3, 2014, pp. 411-426.

Heyward, Will. “Chris Andrews. Interview”. BOMB Magazine, 2012. https://bombmagazine. org/articles/chris-andrews/, consultado 8 de enero 2019.

Hoyos, Héctor. "Bolaño como excusa: contra la representación sinecdótica en la Literatura Mundial”. Letra anexa, no. 1, 2015, pp. 92-106.

Kleinemeier, Marvin. "Ein Interview mit Heinrich von Berenberg”. Wilde Leser, 2009. http://www.wilde-leser.de/?p=1562, consultado 8 de enero 2019.

Locane, Jorge J. De la literatura latinoamericana a la literatura (latinoamericana) mundial. Condiciones materiales, procesos y actores. De Gruyter, 2019.

Loy, Benjamin. "Der Teil der Kritiker: die deutschsprachige Bolaño-Rezeption zwischen Exotismus-Perpetuierung und globaler Prestige-Ökonomie". Roberto Bolaño im deutschsprachigen Kontext. Ed. Stephanie Catani, transcript 2019 (en prensa).

-.-. Roberto Bolaños wilde Bibliothek. Eine Ästhetik und Politik der Lektüre. De Gruyter, 2019.

--.. "The Precarious State of the Art: Writing the Global South and Critical Cosmopolitanism in the Works of J.M. Coetzee and Roberto Bolaño". Re-mapping World Literature. Writing, Book Markets, and Epistemologies between Latin America and the Global South. Eds. Gesine Müller, Jorge J. Locane y Benjamin Loy. De Gruyter, 2018, pp. 91-116.

---. "La ironía como cuestión de Wieder y muerte: Estrella distante de Roberto Bolaño". Ironía y violencia en la literatura latinoamericana. Eds. Brigitte Adriaensen y Carlos van Tongeren. Instituto Internacional de Literatura Iberoamericana, 2018, pp. 189-203.

--.. “Mocking World Literature and Canon Parodies in Roberto Bolaño's Fiction”. Roberto Bolaño as World Literature. Eds. Nicholas Birns y Juan De Castro. Bloomsbury, 2017, pp. 153-166.

--.. "De oposiciones, apropiaciones y traducciones (anti)poéticas: lecturas cruzadas de Nicanor Parra y Roberto Bolaño". La traducción desde, en y hacia Latinoamérica. Perspectivas literarias y lingüísticas. Eds. Silke Jansen y Gesine Müller. Iberoamericana/ Vervuert, 2017, pp. 211-228.

--.. "Chistes par(r)a reordenar el canon - Roberto Bolaño, Nicanor Parra y la poesía chilena”. Romanische Studien, no. 1, 2015, pp. 45-60.

--.. "Deseos de mundo - Roberto Bolaño y la (no tan nueva) literatura mundial”. América Latina y la literatura mundial: mercado editorial, redes globales y la invención de un continente. Eds. Dunia Gras y Gesine Müller. Iberoamericana/Vervuert, 2015, pp. 273-294.

---. “Fantasmas de la periferia o la República Mundial de los Plagios: ‘El viaje de Álvaro Rousselot' de Roberto Bolaño”. Bolaño en sus cuentos. Eds. Paula Aguilar y Teresa Basile. Almenara, 2015, pp. 138-157.

--.. "Escritores bárbaros, detectives distantes y un cura amnésico - escenificaciones de la (post-) dictadura chilena en la obra de Roberto Bolaño". Arpillera sobre Chile. Cine, teatro y literatura antes y después de 1973. Eds. Annette Paatz y Janett Reinstädler. tranvía, 2013, pp. 117-138.

Maldonado Tovar, Juan Camilo. "Escribir a pesar del silencio: una entrevista con Evelio Rosero Diago". El Tiempo, 2017. http://www.eltiempo.com/bocas/una-entrevista-con-el-escritorcolombiano-evelio-rosero-diago-90086, consultado 8 de enero 2019.

Mortati, Julieta. "El actor escondido. Una entrevista con el traductor de Bolaño, Christian Hansen”. alba. lateinamerika lesen, no. 03, 2013, pp. 52-57. 
Pollack, Sarah. “Latin America Translated (Again): Roberto Bolaño’s The Savage Detectives in the United States". Comparative Literature, vol. 61, no. 3, 2009, pp. 346-365.

Sánchez Prado, Ignacio M. “Más allá del mercado. El uso de la literatura latinoamericana en la era neoliberal”. Libro Mercado. Literatura y Neoliberalismo. Ed. José Ramón Ruisánchez Serra. Universidad Iberoamericana, 2015, pp. 15-40.

Spivak, Gayatri Chakravorty. "The Politics of Translation". The Translation Studies Reader. Ed. Lawrence Venuti. Routledge, 2012, pp. 312-330.

---. Death of a Discipline. Columbia University Press, 2003.

Strich, Fritz. Goethe und die Weltliteratur. Francke, 1957.

Valle, José del. "Glotopolítica, ideología y discurso: categorías para el estudio del estatus simbólico del español”. La lengua, ¿patria común? Ideas e ideologías del español. Ed. José del Valle. Iberoamericana/Vervuert, pp. 14-29.

Venuti, Lawrence. "World Literature and Translation Studies". The Routledge Companion to World Literature. Eds. Theo D’haen, David Damrosch y Djelal Kadir. Routledge, 2012, pp. 180-193.

---. The Translator's Invisibility. A History of Translation. Routledge, 2004.

Walkowitz, Rebecca. Born Translated. The Contemporary Novel in an Age of World Literature. Columbia University Press, 2015.

Wilson, Scott Bryan. “The Chris Andrews Interview”. The Quarterly Conversation, 2008. http:// quarterlyconversation.com/the-chris-andrews-interview, consultado 8 de enero 2019. 